\title{
Distinguishing Electric Vehicles from Fossil-Fueled Vehicles with Mobile Sensing
}

\author{
Markus Wüstenberg, Henrik Blunck, Kaj Grønbæk, and Mikkel Baun Kjærgaard \\ Ubiquitous Computing and Interaction, Department of Computer Science \\ Aarhus University, Denmark \\ Email: \{markus,blunck,kgronbak,mikkelbk\}@cs.au.dk
}

\begin{abstract}
Existing methods for transportation mode detection (TMD) using mobile sensing make it generally possible to distinguish between walking, cycling, and motorized transport. However, our means of transport evolve and we develop radically new ways of transporting ourselves; thus new TMD sub-classification methods are needed to distinguish these new transport forms. As we transition from fossil-fueled cars to electric vehicles, switch to bikes with electric motors, ride in hybrid buses, or do city sightseeing on Segways, new challenges arise in distinguishing these from a mobile sensing perspective. Distinguishing electric vehicles (EVs) from fossil-fueled vehicles (FFVs) is a challenge, where traditional methods based on features such as GPS speed, or statistics on raw accelerometer data, are insufficient. In this paper, we present methods for distinguishing EVs from FFVs using smartphones with built-in inertial sensors, by reliably identifying idle-engine motor vibrations through features built on frequency analysis. We provide an extensive analysis of the challenges involved in making the EV/FFV distinction, as well as practical tools based on the methods. This includes analyzing the measurable similarities and differences between EVs and FFVs, and developing methods of reliably separating them. The presented tools implement the methods as classifiers built using machine learning. The analysis of our experiments shows that we can achieve an accuracy of 89-95\% distinguishing EVs from FFVs, even with on-body phones.
\end{abstract}

\section{INTRODUCTION TO ANALYSIS OF ENGINE VIBRATIONS}

Methods for transportation mode detection (TMD) are wellresearched within mobile sensing [1], [2], [3], [4]. There are methods available to distinguish walking, cycling, running, going by car, bus, train, and more, using energy-efficient sensors from everyday mobile phones as a source of input data. Building on this solid foundation of work on TMD in general, this opens up the opportunity to look at TMD from other perspectives. Being interested in forms of transport that impact the environment when used, we turn to creating methods for sub-classification of traveling by car, namely distinguishing electric vehicles (EVs) from fossil-fueled vehicles (FFVs) (see also [5], [6]). This level of distinction is important for example for green accounting, where the environmental impact of a particular transportation mode is used to calculate a total environmental impact of e.g. a fleet of mixed-type cars. The current groups, or classes, as found in the existing literature on TMD are insufficient for doing this. Therefore, currently green accounting is mostly done in a labor-intensive, manual process, where car models and driving specifics are meticulously noted down and impacting factors such as greenhouse gas emissions are subsequently calculated from these data. In contrast, with the distinction methods as proposed in this article, pervasive smartphones used by individuals both privately and at work can be utilized to automatically, easily, and cost-effectively alleviate or maybe even completely remove the burden of this manual process.

In this work, we have compared vibrations sensed through accelerometers in ordinary smartphones in different placements in the car, including on-body phone placements, and developed methods to reliably identify idle-engine motor vibrations through frequency analysis. As a result, independent of where a driver (or passenger) may carry his or her smartphone, we can detect engine vibrations and therefore distinguish an EV from an FFV, with very high accuracy. This form of non-controlled sensing is important to enable people to use these methods in their everyday lives unobtrusively, without having to think about it, nor waste valuable attention better directed elsewhere. While previous work has focused mostly on measurable characteristics during actual transport (such as car acceleration and braking patterns, among other things [2], or various statistical measures [1]), we take a somewhat counterintuitive approach by investigating sensor data in stopped vehicles. This enables us to use our results to broaden our scope: When not relying on phenomena particular to driving cars, but to fossil-fueled combustion engines, the possibility of identifying transport in subways, ferries, tractors, and trucks is within reach. Previous work has also used accelerometers for TMD, but focused on distinction of a broader range of transportation modes, where the classes of FFV and EV were not distinguished but instead represented in one class, either as motorized [1] or as car [2], [3]. For the investigation presented here, we rely on this general classification of distinguishing car travels from other modes of transport; this allows us to focus on sub-classifying vehicles used in car travels into EVs and FFVs.

Apart from the primary goal of presenting a novel method to distinguish EVs from FFVs with mobile sensing using ordinary smartphones, a secondary goal is to better understand the overall goal of accurate, universal transportation mode detection. A better understanding of the challenges involved will aid in making progress towards that broader goal. The difficulty in distinguishing EVs from FFVs lies in the fact that the main consistent difference between an EV and an FFV is in the type of engine. For example, there are cars available that 
have been retrofitted with an electric motor, making this the only distinguishing factor between them and their fossil-fueled siblings.

In order to investigate this difference, we have collected inphone accelerometer data from eight different FFVs and two different EVs, from smartphones placed both on the body (in pockets) and in the car (e.g., in the glove compartment, on the passenger seat), in order to mimic everyday, real-world usage.

Our contributions are:

- A thorough analysis of the challenges involved in distinguishing EVs from FFVs using accelerometers in ordinary smartphones, independent of types of cars as well as phone placement and orientation.

- Concrete machine learning features and tools for accurate separation of the two classes of EV and FFV.

The rest of this paper is structured as follows: In Section II, we delve into the problem of and present our findings on how to distinguish EVs from FFVs. In Section III, we evaluate our approach on real-world datasets and present our results. In Section IV, we discuss our methods, and present potential future challenges as well as opportunities for them. In Section V, we discuss related work, and finally in Section VI, we conclude the paper.

\section{SENSING ENGINE VIBRATIONS IN VEHICLES}

We have developed methods for distinguishing electric vehicles from fossil-fueled vehicles using smartphones with built-in inertial sensors. Instead of relying on measurable phenomena while driving, we analyze the signals from inphone three-axis accelerometers while stopped and are thus able to identify the frequencies of the vibrations emitted by the combustion engine in an FFV. As shown later in the evaluation, this is possible in all tested phone placements both on-body and in the car with very high accuracy, and is furthermore entirely independent of phone orientation. The detection of engine vibrations is stable across different cars, ranging from low-end to high-end models from different manufacturers.

Accelerometers have a low energy profile, making them useful for extended durations of sensing without depleting battery life. As the processing can happen entirely on-phone, no data communication needs to happen, saving further power. This also means that the result does not have to be communicated outside the phone, preserving privacy of transportation mode if needed. Furthermore, online (real-time and on-phone) processing enables the ability to give immediate user feedback, e.g., about current environmental impact [7].

In the following, we will detail our methods for distinguishing EVs from FFVs and compare our methods to existing work.

\section{A. Exploration and analysis of engine vibrations in vehicles}

The vibration of the engine is something you can feel when sitting in an FFV. It might not be a phenomenon we notice much, because we have grown accustomed to sitting in all kinds of vehicles that vibrate in one way or another. EVs do not exhibit this phenomenon, at least not from the engine, and the intuition is therefore that this difference can be detected through inertial sensors, and in particular through accelerometers. Accelerometers have previously been used in TMD, with good results [1], [2], [8]. A few observations are key with respect to sensing engine vibrations with accelerometers:

- Engine vibrations are of a high frequency, compared to acceleration frequencies encountered otherwise while handling a phone. In the case of engine vibrations, we define high-frequency vibrations as vibrations at frequencies above $10 \mathrm{~Hz}$. See Figure 1a for a graph showing the high-frequency vibrations from an idle engine in an FFV, in contrast to the lack of such vibrations in an EV in Figure 1b, both measured by a smartphone in a passenger's trouser pocket. Note that the three axes of the accelerometer have been combined into one acceleration magnitude for this calculation; see details of this calculation below in Section II-C.

- Engine vibrations are quite stable in frequency once the engine is warm and running idle. There is a short interval (typically under five minutes) where some cars show decreasing vibration frequency over time when starting from cold. See Figure 2 for a graph depicting this phenomenon.

- Engine vibrations are (unsurprisingly) linked proportionally to the rotation speed of the engine.

Given these observations, we can show that the vibrations are measurable from three-axis accelerometers in ordinary smartphones. The Figures $1 \mathrm{~b}$ and $1 \mathrm{a}$ depict graphs of multiple frequency analyses of vibrations for an EV and an FFV, respectively. The frequencies are calculated using an FFT [9] on an accelerometer signal at a sample rate of $198 \mathrm{~Hz}$ with a window size of 512 samples, or about 2.6 seconds. The window size is chosen to give a good balance between being able to do the frequency analysis online on the phone with relatively low delay, and at the same time being able to detect sufficiently high frequencies (where the chosen window size gives us a maximum detectable frequency at just under 99 $\mathrm{Hz}$ ). Figure $1 \mathrm{~b}$ shows mostly the frequencies of the inherent sensor noise, but in Figure 1a, on the other hand, clear spikes are visible at the frequencies around $28 \mathrm{~Hz}, 56 \mathrm{~Hz}$, and $78 \mathrm{~Hz}$, which indicates that stable, high-frequency acceleration signals are indeed good separators for distinguishing EVs from FFVs. Note also the smaller spike at around $14 \mathrm{~Hz}$ (or about 840 rpm) in Figure 1a, which, although easily discarded as noise, corresponds quite well with the idle motor rotation speed of just above $800 \mathrm{rpm}$ for this particular car (as read from the gauge in the car). Furthermore, there seems to be a correlation between the frequencies of the peaks, in that they are often (but not always) integer multiples of each other. A more detailed investigation of this is considered future work.

There are, however, also some challenges involved in using these peaks in vibration frequency analyses. We have identified the following challenges, which are addressed by our signal processing, feature design, and application of machine learning: 


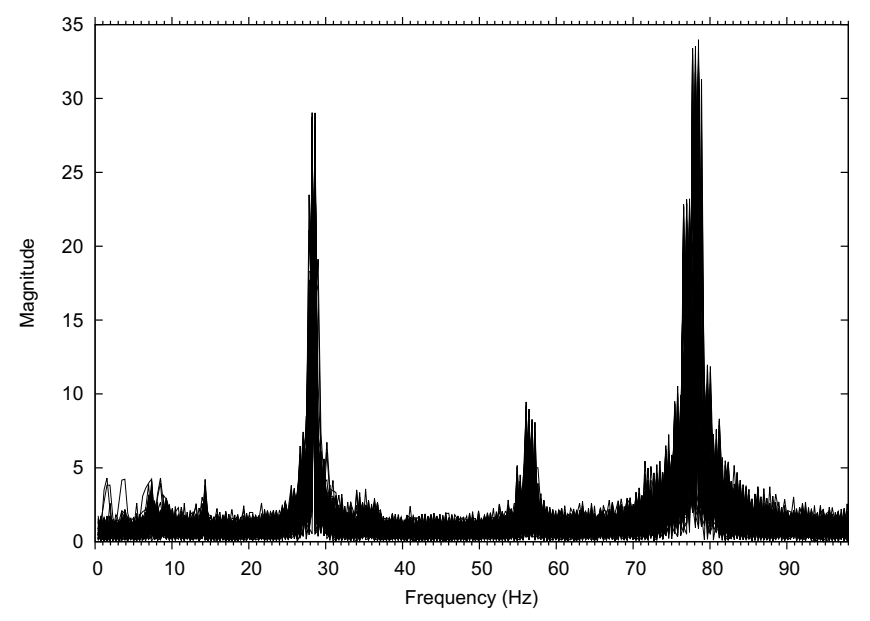

(a) From an FFV.

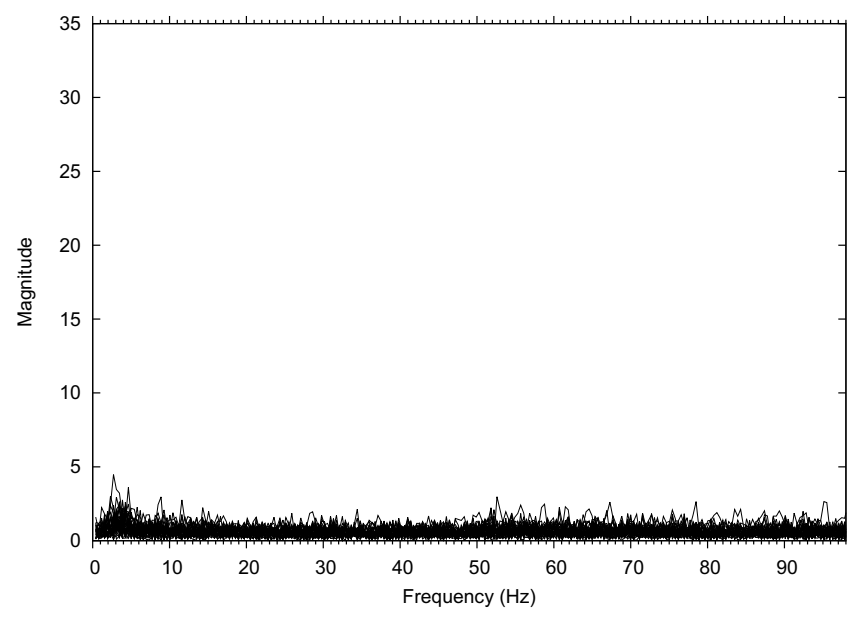

(b) From an EV.

Fig. 1. Frequency analysis of vibrations over several minutes in an FFV (Figure 1a) and an EV (Figure 1b). The phones are placed in the right trouser pocket. Significant, stable peaks are visible at high frequencies in Figure 1a.

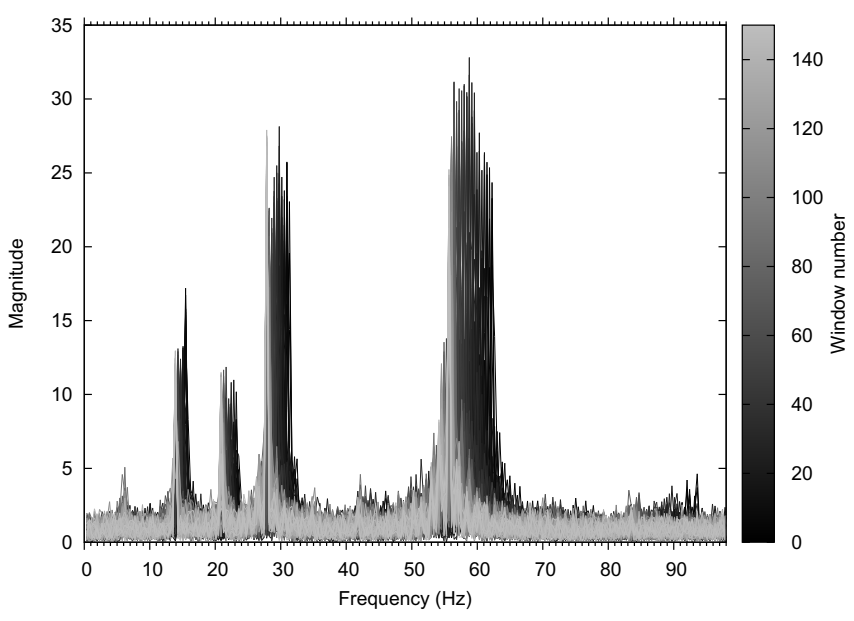

Fig. 2. Frequency analysis of vibrations over about five minutes in an FFV. The phone is placed in the glove compartment. We observe a significant shift of vibration frequencies over time (shown by window number), from higher to lower frequencies.

1) Vibrations can be attenuated or strengthened depending on phone placement. Vibrations from the engine at high frequencies can be dampened when the phone is in a trouser pocket, in a handbag, or just on a cushioned car seat, and can therefore exhibit apparently different high-frequency peaks, making direct comparisons of peaks difficult. Effects of phone placement on TMD accuracy have been examined earlier both for GPS-based [10] and accelerometer-based TMD methods [1]. See Figure 3a, where two different phone placements, one in a trouser pocket and another in the glove compartment, are compared directly. Despite originating from the same experiment, the signals look very different. The peak around $28 \mathrm{~Hz}$ is much larger when measured from the glove compartment than from the trouser pocket, whereas the peak at around $78 \mathrm{~Hz}$ exists only when measured from the pocket.

2) The vibration pattern differs between different kinds of FFVs, transporting and attenuating the engine vibrations differently. High-end cars tend to dampen vibrations better than low-end cars, but on the other hand the former are often equipped with more powerful engines creating stronger vibrations. Figure $3 \mathrm{~b}$ illustrates that the frequency analysis yields quite different results in different FFVs even though the phone placement is the same.

3) When driving, there are several other kinds of vibrations present. Vibrations from the engine are still there (although at different frequencies, depending on the varying rotation speed of the engine), together with vibrations from the physical contact between the car and the road while the car is moving. The external vibrations will also differ significantly based on the phone placement, being dampened in pockets etc., just like the engine vibrations as explained above. To show these external vibrations, frequencies of vibrations from stopped cars in contrast to frequencies of vibrations from driving cars can be seen in Figure 4a for an FFV and in Figure $4 \mathrm{~b}$ for an EV. Note how the vibrations from the engines in the FFV are completely engulfed by external vibrations while driving, and that the magnitude of external vibrations is very similar in FFVs and EVs while driving. Therefore it is easier to look for (a lack of) vibrations caused by a combustion engine while the car in question is stopped.

4) When handling the phone, we disrupt vibrations and change the orientation of the phone in a way that is unpredictable. This can generate low-frequency signals, and implies that we cannot rely on a specific or even static orientation of the phone. 


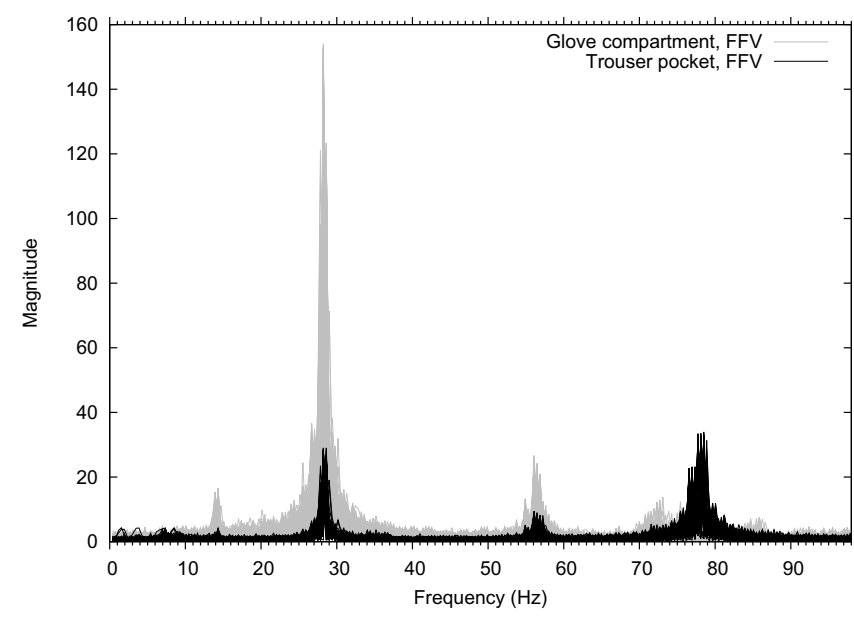

(a) Different placements in the same FFV.

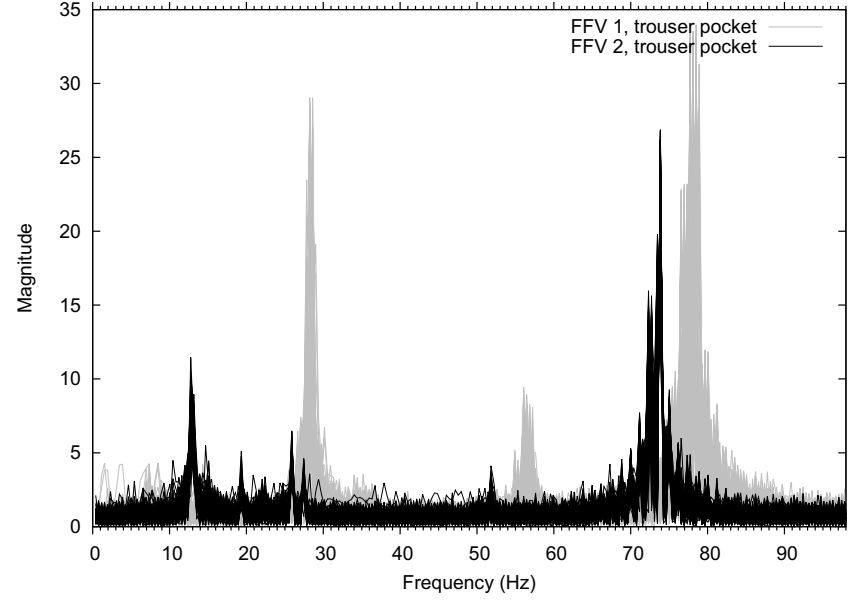

(b) Different FFVs with the same placement.

Fig. 3. Different phone placements have an impact on measured vibrations, and different FFVs exhibit different kinds of vibration frequencies from the same phone placement. Figure 3a shows a frequency analysis on data collected over five minutes in an FFV, with phones placed in a trouser pocket and in the glove compartment. Figure $3 \mathrm{~b}$ shows a frequency analysis of vibrations measured in two different FFVs with phones placed in a trouser pocket both times. Note the different ranges on the value axes.

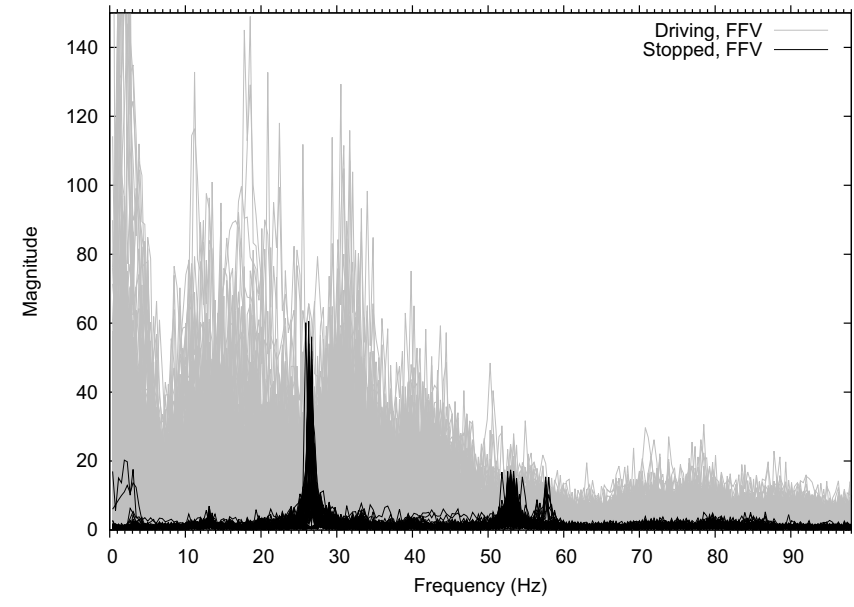

(a) From an FFV.

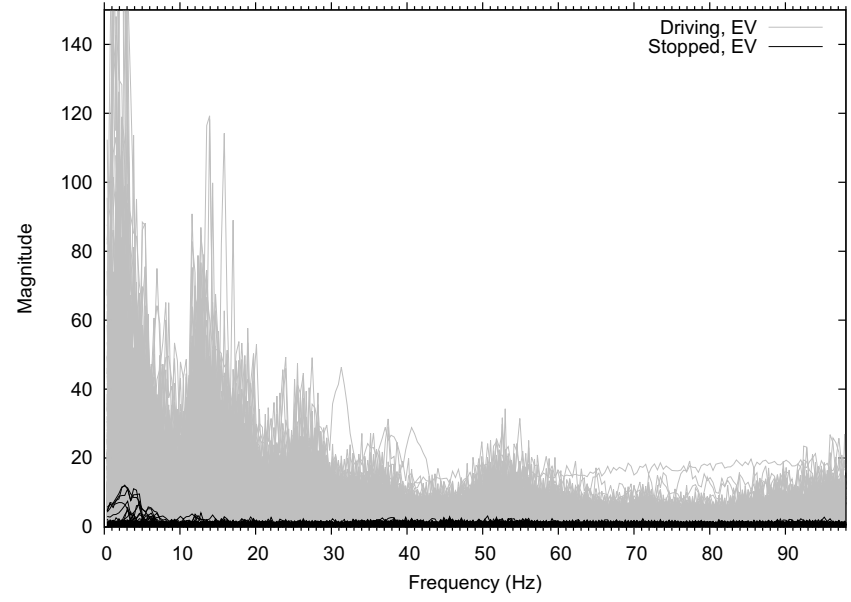

(b) From an EV.

Fig. 4. Graphs showing frequencies of vibrations from stopped cars in contrast to frequencies of vibrations from driving cars, in $4 \mathrm{a}$ from an FFV and in $4 \mathrm{~b}$ from an EV. The phone is placed in the glove compartment.

5) Many newer FFVs automatically turn off the engine when the car is stopped, in order to save energy. This only happens when the engine is warm enough to do so reliably, and is still rather an exceptional feature in cars. Nonetheless, because this feature is gaining popularity, we discuss it in Section IV-B.

Challenges that are not addressed by our methods and which are considered future work are discussed in Section IV.

\section{B. Examining existing TMD methods}

To be able to compare our methods to existing ones, we have analyzed collected accelerometer data with standard statistical features over sliding windows, such as mean, max, standard deviation, and zero crossings [11]. Using these features naively on whole trips of driving produces distinction results no better than random guessing. However, when using accurate stop detection, the above features provide an accuracy of just below $85 \%$ across phone placements during stop periods. While this accuracy may already be useful, we want to further improve accuracies, as well as utilize our domain knowledge (as presented above) to be able to produce features for resolving our exact problem of distinguishing EVs from FFVs. For our use case, green accounting, an error rate of $15 \%$ is still too high to make the approach feasible when competing against the very accurate but manual approach. In contrast, as we show in the evaluation in Section III, the methods we present in this paper almost halve this error rate. We therefore seek to further understand the problem and create features on accelerometer data using the gained domain knowledge. 


\section{Features for machine learning}

Once the differences in high-frequency vibrations between EVs and FFVs are established, we can construct machine learning features to act as separators between the two classes.

First of all, we work on acceleration magnitude data, that is, the signal consists of samples calculated by

$$
A_{\text {magnitude }}=\sqrt{A_{x}^{2}+A_{y}^{2}+A_{z}^{2}}
$$

where $A_{x}, A_{y}$, and $A_{z}$ are the values provided in each sample from a three-axis accelerometer. This makes the signal orientation-independent while preserving frequency information. Secondly, we can discard all low-frequency vibrations below a certain frequency threshold (the cut-off frequency), essentially applying a high-pass filter to our signal. This filters out interference from manual handling of the phones as well as other irrelevant signals, including those from movements of the body, while keeping the relevant high-frequency vibration signals. However, there might still be interfering vibration signals in special cases, see Section IV-D. The cut-off frequency was empirically established and set at $19 \mathrm{~Hz}$. Thirdly, to altogether remove the impact of vibrations caused by driving, we simply only work on acceleration signals when the car is stopped. The underlying assumption is that cars stop at least once during a trip, e.g., at an intersection with a red light or, for the unlucky, maybe in a traffic jam. Detecting stops is certainly possible, and has been proposed using different means: Hemminki et al. [2] employ a stationary classifier which analyzes horizontal acceleration and braking patterns over time, and Kjærgaard et al. [12] analyze the total acceleration energy across all frequencies and also make use not only of accelerometer but also of GPS speed and location data.

Favoring a simple approach, both for the sake of of savings in processing power on resource-constrained devices, as well as for the intrinsic value of simplicity (see [13], [14]), we choose a small set of features. The variable parameters are the noise floor threshold and the cut-off frequency. In our case, we use two different noise floor thresholds, ending up with two sets of these three features:

- peaks_exist: A boolean value stating if there are highfrequency vibration peaks above the noise floor threshold at all. The noise floor threshold is set empirically for each phone/sensor type, on a stationary phone.

- percentage_of_values_above_noise_floor: A percentage of frequency values above the noise floor threshold.

- highest_peak_value: The value of the highest frequencydomain peak.

As shown in the evaluation, these features are sufficient to achieve a high accuracy in distinguishing EVs from FFVs. Other, more sophisticated features were considered as well, such as identifying signal peaks at specific frequencies. Because of the variables involved (phone placement and different models of cars), however, it is hard to anticipate where these signal peaks might be, and therefore identify them. We consider it future work to find vibration frequency commonalities sufficiently stable to work across phone placements and car models to develop more sophisticated features that can further improve accuracy.

\section{Performance eVAluation of ML FeAtures}

In this section we introduce our data collection method for training and test data, and evaluate the performance of our analysis methods on real-world driving data.

\section{A. Data collection for training and test}

For training our algorithms, we have collected data from eight different FFVs, ranging from low-end to high-end models, as well as from two different EVs. According to our observations, different EVs are very similar with regard to our analyses, because no high-frequency vibrations from a combustion engine are present. Therefore, it is more important for us to study data from a broad range of FFVs, in order to challenge our methods and investigate whether their performance is generalizable across different FFVs. For training data, we are using mostly data that was collected in cars standing still with the engine on, whereas for test data we solely use real-world driving data collected while driving in an urban environment with frequent stops at intersections, turns, etc. Test data from trips on highways, for example, would yield fewer stops during which to perform classification, but would otherwise not impact analysis results. The reasoning is that a trip has to start in a non-driving state, stop in a non-driving state, and more often than not have stops at some point during the trip, providing the necessary classification time windows.

We argue that when using accurate stop detection, the realworld data and the data collected in idle cars are interchangeable. For the real-world test datasets, stops (of 5 seconds or longer) are manually labeled, in contrast to using automatic stop detection. The impact of an inaccurate stop detection on overall accuracy is discussed in Section IV-A. There is of course no overlap between training and test data.

For each car, six different phone placements are considered. The on-body placements are left and right trouser pockets (both in relatively tight jeans and loose trousers) and in a loose jacket pocket, see Figure 5a. The in-car placements are a phone placed near the gear shift, one placed on the passenger seat, and finally one in the glove compartment, see Figure 5b. Test data are collected from slightly different placements, but as we only distinguish between on-body and in-car placements in our evaluation, this has no impact on the results. Data was collected with LG Nexus 4 smartphones running Android 4.3 and later Android 4.4, by the authors and colleagues. This is a relatively new high-end smartphone with modern hardware, and in particular it has an accelerometer with a high sample rate of just below $200 \mathrm{~Hz}$. According to the Nyquist-Shannon sampling theorem, this enables us to detect frequencies up to a limit of just below $100 \mathrm{~Hz}$. Other smartphones, and in particular older phones, might not have as high a sample rate, and some high frequencies might thus be undetectable on these. However, many of the detected vibration peaks are much below $100 \mathrm{~Hz}$, which means they could be detected on phones with accelerometers with a lower sample rate. A more 


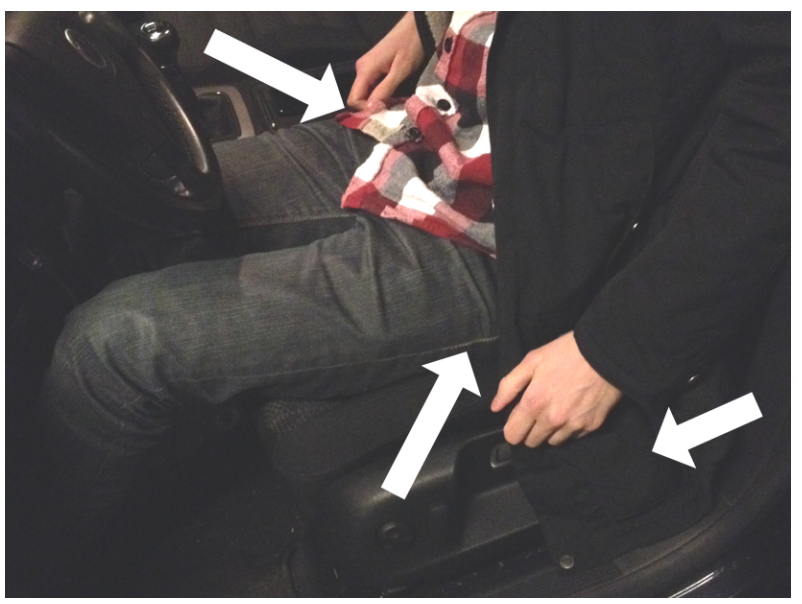

(a) On-body phone placements.

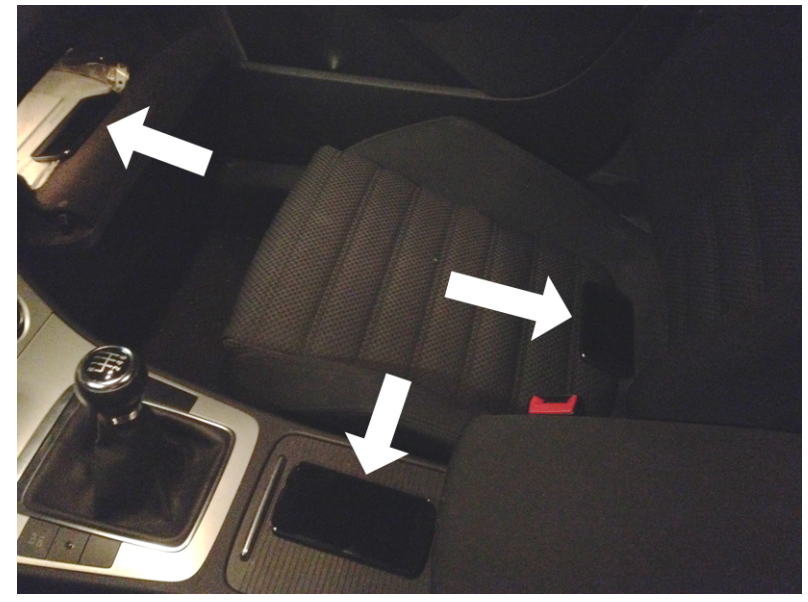

(b) In-car phone placements.

Fig. 5. Phone placements.

detailed investigation of these challenges is considered future work. We have highlighted heterogeneity issues like these in earlier work, see [15].

\section{B. Accuracy distinguishing EVs from FFVs}

To evaluate the accuracy of distinguishing EVs from FFVs, we investigate both the overall accuracy as well as accuracy per phone placement: we distinguish between on-body (that is, in various pockets) or in-car (on the seat, glove compartment, etc.) placements. Furthermore, we investigate how detection accuracy is impacted when looking at accuracy per stop and per trip. In related work, often the performance measures precision and recall are used (see e.g., [1], [2]). We only report the accuracy measure here, since the precision and recall values in our case are similar or identical.

We are using standard machine learning algorithms to build classifiers on our features. The implementations are taken from the Weka machine learning toolset [16]. In Table I, we give an overview of the accuracies achieved with different learners, both for 10-fold 10-times cross-validated data, and with independent test sets across phone placements, as well as for only in-car and for only on-body placements, respectively, as described earlier. The results are accuracies across the whole test dataset-a short discussion on per-stop accuracy is given below. Note that the cross-validated data is overly optimistic, but added here for completeness and comparison to other papers, e.g., Reddy et al. [1].

Our overall accuracy distinguishing EVs from FFVs, across phone placements, is $91.6 \%$ with separate training and test sets. This means that we can detect engine vibrations from whereever the phone is placed in the car with very high accuracy. The overall accuracy for only in-car phones is $95.6 \%$, and the overall accuracy for on-body phones is slightly lower, at $89.5 \%$. These results are gained using training data that have not been resampled, and therefore contain more instances collected in FFVs than EVs. Resampling with bias towards uniformity, both with and without replacement, produces very

\begin{tabular}{lrrrr}
\hline Classifier & Cross-validated & All placements & In-car & On-body \\
\hline OneR & 99.1 & 90.9 & 92.5 & 89.0 \\
Random Forest & 98.9 & $\mathbf{9 1 . 6}$ & 93.7 & 89.2 \\
Decision Tree & $\mathbf{9 9 . 2}$ & 90.7 & 92.5 & 88.7 \\
BayesNet & 92.9 & 81.9 & $\mathbf{9 5 . 6}$ & 68.3 \\
SVM & $\mathbf{9 9 . 2}$ & 91.0 & 92.2 & $\mathbf{8 9 . 5}$ \\
kNN & 98.8 & 90.0 & 92.9 & 86.8 \\
\hline
\end{tabular}

TABLE I

CLASSIFICATION RESULTS FOR DIFFERENT CLASSIFIERS, ALL TRAINING DATA. NUMBERS ARE PERCENTAGES OF CORRECTLY CLASSIFIED INSTANCES.

\begin{tabular}{lrrrr}
\hline Classifier & Cross-validated & All placements & In-car & On-body \\
\hline OneR & 96.4 & $\mathbf{9 1 . 2}$ & 95.7 & $\mathbf{8 6 . 6}$ \\
Random Forest & 96.2 & 91.1 & $\mathbf{9 6 . 9}$ & 85.2 \\
Decision Tree & 96.7 & 90.6 & 96.8 & 84.4 \\
BayesNet & 94.1 & 78.7 & 93.2 & 64.1 \\
SVM & $\mathbf{9 7 . 2}$ & 90.5 & 96.8 & 84.3 \\
kNN & 95.9 & 89.2 & 96.5 & 81.9 \\
\hline
\end{tabular}

TABLE II

CLASSIFICATION RESULTS FOR DIFFERENT CLASSIFIERS, TRAINING DATA IS RESAMPLED WITHOUT REPLACEMENT FOR UNIFORM DISTRIBUTION OF EACH CLASS. NUMBERS ARE PERCENTAGES OF CORRECTLY CLASSIFIED INSTANCES.

\begin{tabular}{lrrrr}
\hline Classifier & Cross-validated & All placements & In-car & On-body \\
\hline OneR & 99.2 & 90.4 & 95.7 & 85.0 \\
Random Forest & 99.7 & $\mathbf{9 1 . 8}$ & 94.7 & $\mathbf{8 8 . 7}$ \\
Decision Tree & 99.5 & 91.4 & 94.9 & 87.7 \\
BayesNet & 96.5 & 79.9 & 95.0 & 64.8 \\
SVM & 97.7 & 90.9 & $\mathbf{9 7 . 4}$ & 84.6 \\
kNN & $\mathbf{9 9 . 8}$ & 90.1 & 93.8 & 86.2 \\
\hline
\end{tabular}

TABLE III

CLASSIFICATION RESULTS FOR DIFFERENT CLASSIFIERS, TRAINING DATA IS RESAMPLED WITH REPLACEMENT FOR UNIFORM DISTRIBUTION OF EACH CLASS. NUMBERS ARE PERCENTAGES OF CORRECTLY CLASSIFIED INSTANCES. 
similar results, as seen in Tables II and III.

The results presented above are percentages over the whole test dataset. However, it is also interesting to evaluate accuracies as measured per-stop and per-trip. Especially when stops are short, only a few windows of data will be analyzed and classified. Even with a high accuracy, misclassifications will happen, and with few windows per stop, data from a single stop could suggest a different class of car. To alleviate this problem, we can look at whole trips, and classify a trip as being in an EV or FFV based on the classification of its stops. To counter misclassifications based on short stops, either the minimum needed stop length could be adjusted, or weights added based on stop duration, as the chance of misclassification per stop is expected to drop when more windows are available for classification. Doing this form of trip-based post-processing could land us at the magic accuracy of $100 \%$ across phone placements, if not for a single trip consistently misclassifying; see below.

\section{Consistent error sources}

One large source of error in our test dataset is a single trip in an FFV with phone placements in two trouser pockets. For reasons yet to be identified, the vibration frequency signal is attenuated to a point where it is almost indistinguishable from the noise, i.e. the signal-to-noise ratio (SNR) is very low. When comparing plots of these data visually with plots of data from other placements from the same trip, it is easy to see where the peaks are, although they are very small. When removing this only really problematic trip from the test dataset, our overall accuracy jumps to $95.5 \%$. It is important to note that this form of consistent errors in classification is hard to correct, even in post-processing, and the possibility of this happening is something we should still be aware of even at high accuracies. We can of course not just remove test data to improve our results, edge-case or not, so this anomaly is left as-is.

\section{DISCUSSION OF CHALLENGES AND OPPORTUNITIES}

In this section, we will discuss further potential challenges for distinguishing EVs from FFVs in real-world sensing situations, as well as opportunities for using our distinction methods in other areas of transportation mode detection.

\section{A. Impact of inaccurate stop detection}

In our distinction methods, we have relied on accurate stop detection in order to detect engine vibrations. We have given examples of how this can be done, but have chosen not to implement this ourselves, opting instead to manually label stops in our training and test datasets. In this section, we discuss the impact of inaccurate stop detection on the detection accuracy.

We start out by pointing out an important observation, namely that speeds are low when coming to a stop or when accelerating just after a stop. This works in our favor, as external high-frequency vibrations are more prevalent at higher speeds. With this in mind, we can look at Figures $6 a$ and $6 b$,

\begin{tabular}{lrr}
\hline Test method & Additional instances & Accuracy \\
\hline Accurate test set & $0 \%$ & $91 \%$ \\
Inaccurate test set, 5 second padding & $34 \%$ & $88 \%$ \\
Inaccurate test set, 10 second padding & $67 \%$ & $81 \%$ \\
Inaccurate test set, 15 second padding & $100 \%$ & $78 \%$ \\
\hline
\end{tabular}

TABLE IV

CLASSIFICATION RESULTS FOR TEST SETS BUILT WITH INACCURATE STOP DETECTION.

which show the effects of inaccurate stop detection on the frequency analysis. We see that significant noise from external vibrations is present, but less than when compared with that seen in Figure 4.

To evaluate the impact more formally, we have evaluated one of our classifiers on different test sets created with deliberately inaccurate stop detection. In each of these test sets, the begin and end times of stops have been padded in both directions with 5, 10, and 15 more seconds of data compared to the accurate test set, totalling 10, 20, and 30 seconds more data for each stop. To clarify, each of these inaccurate test sets is a superset of the accurate test set, with some extra data added from the driving just before each stop and from just after each stop. The classification results can be seen in Table IV, along with percentages of how many more instances are added to each test set compared to the accurate test set. With a 5 second extra data padding at the beginning and end of each stop, the accuracy drops three percentage points, to $88 \%$, and with a worst-case padding of 15 seconds at each stop, accuracy drops to $78 \%$ correctly classified instances. This indicates that the impact of inaccurate stop detection on overall distinction accuracy is manageable.

\section{B. Automatic engine shut-off}

Newer FFVs sometimes come equipped with a feature that turns off the engine when the car is stopped, e.g., at a traffic light. This can make it difficult to detect engine vibrations when the car is stopped, as there might be none. However, initial investigations show that engine vibrations can still be detected with fairly high accuracy at slow speeds just before and after stops, where the automatic engine shut-off feature is not yet or not anymore in effect. This is similar to the analysis of effects of inaccurate stop detection described above, except that only data from just before and just after stops are included, not from the actual stop. Furthermore, the shut-off feature is usually only enabled when the engine is sufficiently warm, in order to make sure that it starts immediately when needed. This means that engine vibrations can be detected at the beginning of a trip, when the engine is still relatively cold and the feature is still disabled. Finally, we have shortly experimented with detecting the distinct event of turning on and off the engine, which can "shake" the whole car measurably. Designing and evaluating a suitable pattern recognition for detecting this phenomenon seems promising, and we consider its further investigation in future work. 


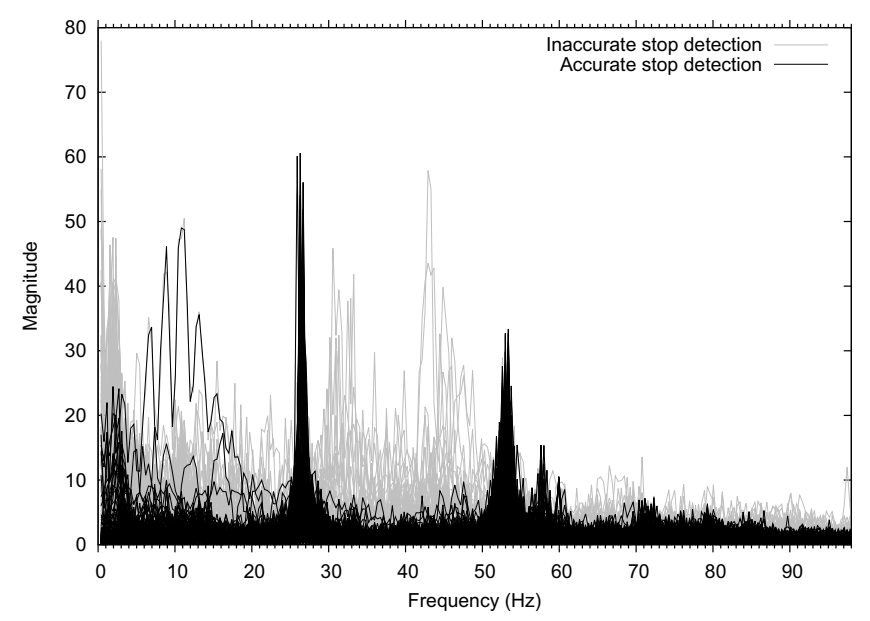

(a) From an FFV.

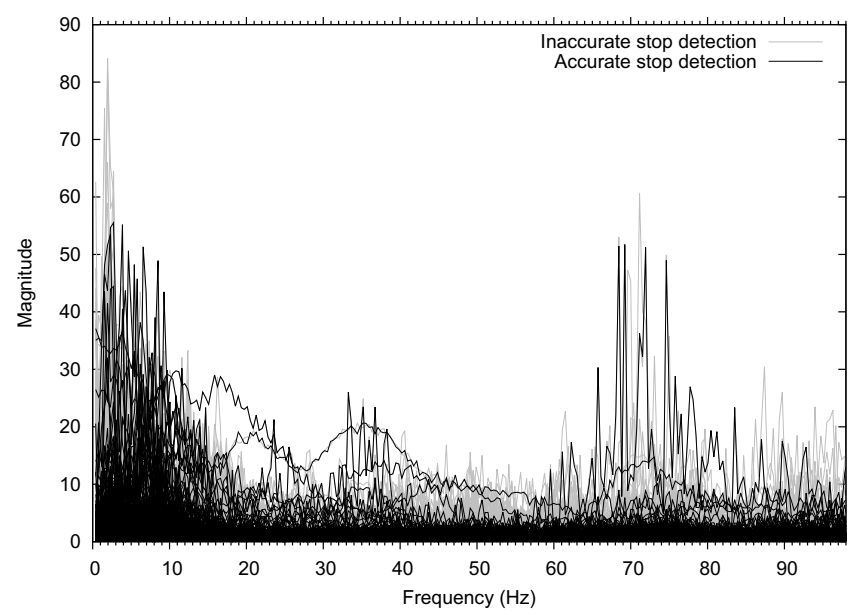

(b) From an EV.

Fig. 6. Frequency analysis of vibrations with both accurate and inaccurate stop detection overlaid, for an FFV (Figure 6a) and an EV (Figure 6b). This includes all phone placements. The inaccurate stop detection padding is 5 seconds at each end.

\section{Hybrid electric vehicles}

We are faced with an interesting problem when so-called hybrid electric vehicles are involved, which are equipped with both an electric and a fossil-fueled engine, which can be automatically switched between by the car to optimize energy efficiency, even while driving. It is interesting both because it challenges our detection methods, but also because the environmental impact of hybrid electric vehicles is much more difficult to derive manually for example for the sake of green accounting. For an accurate representation of environmental impact, the switches between engines should be recorded for subsequent emission calculations. Unfortunately, this is not currently possible with our classification methods, as we rely on a stopped vehicle when classifying, but it could be possible to detect if a hybrid electric vehicle is being used. Although we have not tested our detection methods with hybrid electric vehicles, we can discuss some expected challenges.

A hybrid electric vehicle, such as the Toyota Prius, usually needs some time to warm up the fossil-fueled engine at the beginning of a trip before the engine can be switched on and off frequently, parallel to what is discussed in the section about automatic engine shut-off above. This would probably leave enough time to utilize the detection methods, given that the car is stopped at some point during these first minutes. After this, however, it would get harder to do the distinction, as the fossil-fueled engine is often turned off at lower speeds and at stops, giving no opportunity to sense engine vibrations. Hybrid electric vehicles therefore give a good reason for exploring methods for detecting vibrations from fossil-fueled engines also when the car is moving, and we consider this very relevant future work.

\section{Other sources of vibrations}

Other sources of vibrations might impact the distinction accuracy, so it makes sense to briefly consider what these might be in ordinary cars. One that comes to mind is a car's sound system. Because we are looking at frequencies in the audible spectrum (above roughly $20 \mathrm{~Hz}$ ), car speakers can produce sounds with frequencies in that range, and more importantly also create vibrations in the materials of the car by the electromagnetically induced movement of the speaker itself. Preliminary experiments show that very high volumes of music can indeed be picked up by the accelerometer, but very dependent on phone placement: where phones on the body barely pick up a signal, phones in the glove compartment sense vibrations from the surface they are placed on. The power output of the sound system and speakers, as well as speaker size, likely are also relevant variables. Contrary to the vibrations from the engine, for standard quality audio systems these vibration signals from music are both of a higher frequency (depending on the frequency range of the car speakers and the audio signal), and much more variable, which indicates that it is possible to filter these out.

Another source of vibrations to consider is the air conditioning system often built into cars. Again, initial investigations show that vibrations from fans running at full speed etc. can be picked up by the accelerometer, but exclusively at in-car phone placement, assumedly because these are of a much lower magnitude than vibrations from a fossil-fueled engine. Contrary to the vibrations from the music, these vibrations are very stable in frequency and can be at the same low frequencies as engine vibrations. Given that phone placement is unknown, it could prove challenging to distinguish e.g., vibrations from an $\mathrm{A} / \mathrm{C}$ sensed from a phone placed in the glove compartment, from vibrations from a fossil-fueled engine sensed from a phone placed in a trouser pocket. Using more domain knowledge of the frequencies of idle engines (often around 800-1000 rpm) in the machine learning feature design might prove useful in this regard. Both this, and the filtering of vibrations from the sound system, is considered future work. 


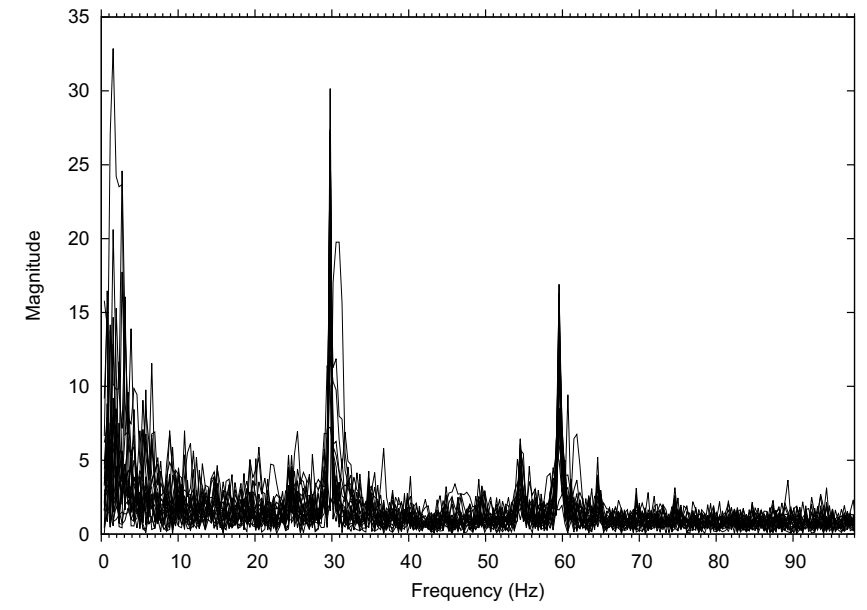

Fig. 7. Frequency analysis of vibrations at bus stops in a bus. The phone is placed in the left trouser pocket. Significant high-frequency peaks are present.

\section{E. Opportunities in broader transportation mode detection}

Although our primary goal is to distinguish EVs from FFVs, our methods can be employed to improve other areas of TMD as well. What we have presented is in effect a general method to detect engine vibrations from smartphones placed anywhere in a car. Conversely, this means that we can also, with quite high accuracy, detect the absence of engine vibrations in this form. This could be used to improve accuracy in other modes of transport: to distinguish driving a car or a bus from a Segway, detecting no engine vibrations when going downhill on a bike and thus ruling out that possibility, detecting being on a ferry (its huge engines placed elsewhere within its hull), or distinguishing between an electric and a fossil-fueled metro ride, to measure environmental impact.

As an example, consider Figure 7, where we have briefly investigated engine vibrations as measured by smartphones in a bus. Even when the engine is several meters away, the engine vibrations are still quite clear, indicating that this kind of vibration analysis can be successfully employed in other transportation modes.

\section{RELATED WORK}

The work presented here relates to work within the fields of transportation mode detection and vehicular classification. Transportation mode detection has been an increasingly active research topic specifically within the ubiquitous computing community. Most related recent publications within the community focus on the application of mobile sensing [17], utilizing modern user devices such as smartphones (and their built-in motion and location sensors) as data sources for inferring the user's transportation mode. Specifically, Reddy et al. [1] compare the impact of different combinations of smartphone sensors, namely an accelerometer, GPS, WiFi, and GSM, on detection accuracy and energy consumption, when distinguishing between basic transport modes, namely between walking, running, biking and motorized transport. Reddy et al. conclude that the combination of the first two sensors provides the best trade-off.

Further transportation mode detection systems have been presented which rely either solely on GPS data, e.g., by Zheng et al. [4], Liao et al. [18], and Bolbol et al. [19], or on accelerometer data, e.g., by Wang et al. [8] and later Hemminki et al. [2]. In a comparative evaluation Hemminki et al. concluded that their system outperforms those from Wang et al. and Reddy et al. in terms of accuracy. Furthermore, recent systems, e.g., by Hemminki et al., Zheng et al., Wang et al., and Bolbol et al., aim to distinguish also between different motorized modes of transport such as car, bus, metro, and tram. Stenneth et al. [3] achieve such a distinction relying on GPS location data and a correlation of such data with bus and train schedules.

A subdivision in different motorized transportation modes as addressed by Hemminki et al. and Stenneth et al. is crucial for transportation mode detection, e.g., when it is used for green accounting or eco-feedback provisions [5], [7], since motorized transportation choices, such as fossil-fueled car vs. train travel, come with vastly different environmental impacts; the same holds for fossil-fueled vs. electric car transportation [20], [21].

Thus, the investigations in this paper address a need in transportation mode distinguishing which is - to the best of our knowledge_-not yet addressed in related work, neither within the transportation mode detection literature nor within the field of vehicle classification. In the latter, most solutions rely on instrumentation of vehicles with dedicated sensors or, more commonly, fixed-location sensing installations, utilizing e.g., cameras, loop detectors, piezoelectric or magnetic sensors [22]. Recently, however, Sun and Ban [23] utilized mobile GPS data, as can be collected by smartphones, for vehicle classification; they restrict themselves though to the binary classification problem of distinguishing passenger cars from trucks. The distinction of electric, fuel-cell, and fossil-fuel cars as focused on in this paper, may be of importance for some of the prominent application areas of vehicle classification including traffic and fleet management and facility planningdue to the different maximal ranges and fueling needs of these vehicle types.

\section{CONCLUSION}

In this paper, we have presented a method to detect engine vibrations in FFVs to distinguish these from EVs with an overall $91.6 \%$ accuracy. We have characterized engine vibrations from FFVs as being stable in frequency (visible in a frequency analysis at a few distinct peaks) and linked proportionally to the rotation speed of the engine. The challenges to their detection are primarily that they can be altered based on the type of car and the phone placement and orientation. Engine vibrations are hard to measure when driving, which is why we attempt classification only when the car is stopped. However, even moderately inaccurate stop detection, by a matter of 515 seconds, degrades the accuracy only somewhat, from 3-13 percentage points. We evaluated our simple machine learning 
features on a dataset collected from eight FFVs and two EVs across many different phone placements.

This type of distinction can now be used for automatic collection of information for green accounting purposes, making it more attractive for companies to do so easily and costeffectively, and without putting extra burdens on the people doing the data collection, freeing them to do their actual job. It can also be used as a general engine detection method, used to improve robustness of TMD in general. These kinds of accessible tools could then be part of steering the future of and possibilities within green accounting, in the end benefiting both the companies using them, the people influenced by them, and the environment in general.

\section{ACKNOWLEDGMENT}

This work has been supported by The Danish Council for Strategic Research as part of the EcoSense project (11115331).

\section{REFERENCES}

[1] S. Reddy, M. Mun, J. Burke, D. Estrin, M. Hansen, and M. Srivastava, "Using mobile phones to determine transportation modes," ACM Trans. Sen. Netw., vol. 6, no. 2, pp. 13:1-13:27, Mar. 2010. [Online]. Available: http://doi.acm.org/10.1145/1689239.1689243

[2] S. Hemminki, P. Nurmi, and S. Tarkoma, "Accelerometer-based transportation mode detection on smartphones," in Proceedings of the 11th ACM Conference on Embedded Networked Sensor Systems, ser. SenSys '13. New York, NY, USA: ACM, 2013, pp. 13:1-13:14. [Online]. Available: http://doi.acm.org/10.1145/2517351.2517367

[3] L. Stenneth, O. Wolfson, P. S. Yu, and B. Xu, "Transportation mode detection using mobile phones and gis information," in Proceedings of the 19th ACM SIGSPATIAL International Conference on Advances in Geographic Information Systems, ser. GIS '11. New York, NY, USA: ACM, 2011, pp. 54-63. [Online]. Available: http://doi.acm.org/10.1145/2093973.2093982

[4] Y. Zheng, Y. Chen, Q. Li, X. Xie, and W.-Y. Ma, "Understanding transportation modes based on gps data for web applications," $A C M$ Trans. Web, vol. 4, no. 1, pp. 1:1-1:36, Jan. 2010. [Online]. Available: http://doi.acm.org/10.1145/1658373.1658374

[5] J. Froehlich, T. Dillahunt, P. Klasnja, J. Mankoff, S. Consolvo, B. Harrison, and J. A. Landay, "Ubigreen: Investigating a mobile tool for tracking and supporting green transportation habits," in Proceedings of the SIGCHI Conference on Human Factors in Computing Systems, ser. CHI '09. New York, NY, USA: ACM, 2009, pp. 1043-1052. [Online]. Available: http://doi.acm.org/10.1145/1518701.1518861

[6] M. Mun, S. Reddy, K. Shilton, N. Yau, J. Burke, D. Estrin, M. Hansen, E. Howard, R. West, and P. Boda, "Peir, the personal environmental impact report, as a platform for participatory sensing systems research," in Proceedings of the 7th International Conference on Mobile Systems, Applications, and Services, ser. MobiSys '09. New York, NY, USA: ACM, 2009, pp. 55-68. [Online]. Available: http://doi.acm.org/10.1145/1555816.1555823

[7] J. Froehlich, L. Findlater, and J. Landay, "The design of ecofeedback technology," in Proceedings of the SIGCHI Conference on Human Factors in Computing Systems, ser. CHI '10. New York, NY, USA: ACM, 2010, pp. 1999-2008. [Online]. Available: http://doi.acm.org/10.1145/1753326.1753629

[8] S. Wang, C. Chen, and J. Ma, "Accelerometer based transportation mode recognition on mobile phones," in 2010 Asia-Pacific Conference on Wearable Computing Systems (APWCS), Apr. 2010, pp. 44-46.

[9] J. W. Cooley and J. W. Tukey, "An algorithm for the machine calculation of complex fourier series," Mathematics of Computation, vol. 19, no. 90, pp. pp. 297-301, 1965. [Online]. Available: http://www.jstor.org/stable/2003354
[10] H. Blunck, M. B. Kjærgaard, and T. S. Toftegaard, "Sensing and classifying impairments of gps reception on mobile devices," in Pervasive Computing, ser. Lecture Notes in Computer Science, K. Lyons, J. Hightower, and E. Huang, Eds. Springer Berlin Heidelberg, 2011, vol. 6696, pp. 350-367. [Online]. Available: http://dx.doi.org/10.1007/978-3-642-21726-5_22

[11] D. Figo, P. Diniz, D. Ferreira, and J. Cardoso, "Preprocessing techniques for context recognition from accelerometer data," Personal and Ubiquitous Computing, vol. 14, no. 7, pp. 645-662, 2010. [Online]. Available: http://dx.doi.org/10.1007/s00779-010-0293-9

[12] M. B. Kjærgaard, J. Langdal, T. Godsk, and T. Toftkjær, "Entracked: Energy-efficient robust position tracking for mobile devices," in Proceedings of the 7th International Conference on Mobile Systems, Applications, and Services, ser. MobiSys '09. New York, NY, USA: ACM, 2009, pp. 221-234. [Online]. Available: http://doi.acm.org/10.1145/1555816.1555839

[13] R. Holte, "Very simple classification rules perform well on most commonly used datasets," Machine Learning, vol. 11, no. 1, pp. 63-90, 1993. [Online]. Available: http://dx.doi.org/10.1023/A\%3A1022631118932

[14] P. Domingos, "A few useful things to know about machine learning," Commun. ACM, vol. 55, no. 10, pp. 78-87, Oct. 2012. [Online]. Available: http://doi.acm.org/10.1145/2347736.2347755

[15] H. Blunck, N. O. Bouvin, T. Franke, K. Grønbæk, M. B. Kjærgaard, P. Lukowicz, and M. Wüstenberg, "On heterogeneity in mobile sensing applications aiming at representative data collection," in Proceedings of the 2013 ACM Conference on Pervasive and Ubiquitous Computing Adjunct Publication, ser. UbiComp '13 Adjunct. New York, NY, USA: ACM, 2013, pp. 1087-1098. [Online]. Available: http://doi.acm.org/10.1145/2494091.2499576

[16] M. Hall, E. Frank, G. Holmes, B. Pfahringer, P. Reutemann, and I. H. Witten, "The weka data mining software: An update," SIGKDD Explor. Newsl., vol. 11, no. 1, pp. 10-18, Nov. 2009. [Online]. Available: http://doi.acm.org/10.1145/1656274.1656278

[17] N. Lane, E. Miluzzo, H. Lu, D. Peebles, T. Choudhury, and A. Campbell, "A survey of mobile phone sensing," Communications Magazine, IEEE, vol. 48, no. 9, pp. 140-150, 2010.

[18] L. Liao, D. J. Patterson, D. Fox, and H. Kautz, "Learning and inferring transportation routines," Artificial Intelligence, vol. 171, no. 5-6, pp. 311-331, 2007. [Online]. Available: http://www.sciencedirect.com/science/article/pii/S0004370207000380

[19] A. Bolbol, T. Cheng, I. Tsapakis, and J. Haworth, "Inferring hybrid transportation modes from sparse GPS data using a moving window SVM classification," Computers, Environment and Urban Systems, vol. 36, no. 6, pp. 526-537, Nov. 2012. [Online]. Available: http://www.sciencedirect.com/science/article/pii/S0198971512000543

[20] C. E. Thomas, "Fuel cell and battery electric vehicles compared," International Journal of Hydrogen Energy, vol. 34, no. 15, pp. 6005-6020, Aug. 2009. [Online]. Available: http://www.sciencedirect.com/science/article/pii/S0360319909008696

[21] E. Holden and K. G. Hyer, "The ecological footprints of fuels," Transportation Research Part D: Transport and Environment, vol. 10, no. 5, pp. 395-403, Sep. 2005. [Online]. Available: http://www.sciencedirect.com/science/article/pii/S1361920905000271

[22] L. E. Y. Mimbela and L. A. Klein, "Summary of vehicle detection and surveillance technologies used in intelligent transportation systems," 2000. [Online]. Available: http://trid.trb.org/view.aspx?id=681316

[23] Z. Sun and X. J. Ban, "Vehicle classification using GPS data," Transportation Research Part C: Emerging Technologies, vol. 37, pp. 102-117, Dec. 2013. [Online]. Available: http://linkinghub.elsevier.com/retrieve/pii/S0968090X13002040 